\title{
Study of the high-energy diffuse neutrino signal with the ANTARES telescope
}

\author{
The ANTARES Collaboration ${ }^{\ddagger *}$ \\ $\$$ full author list at PoS(ICRC2019)1177 \\ E-mail: luigi.fuscodapc.in2p3.fr
}

\begin{abstract}
The analysis of the ANTARES all-flavour 9-year data sample provided the observation of a mild excess of events over the expected atmospheric backgrounds. This excess is consistent with the high-energy diffuse cosmic neutrino signal detected by the IceCube Neutrino Observatory, using different analysis strategies and data sample, though less significant because of the smaller detector size. Nevertheless, the analysis of ANTARES data can provide valuable information in the study of the high-energy neutrino signal. The data sample of the previously published analysis has been expanded adding $\sim 900$ additional days of detector data acquisition time. The results of the analysis of this additional set are reported in this contribution. A $1.8 \sigma$ excess above the expectations from atmospheric backgrounds is observed in data. The null-cosmic contribution in the high-energy sample is excluded at $90 \%$ confidence level.
\end{abstract}

\footnotetext{
Corresponding authors: Luigi Antonio Fusco ${ }^{\dagger 1}$, Federico Versari ${ }^{2}$

${ }^{1}$ APC, Univ Paris Diderot, CNRS/IN2P3, CEA/Irfu, Obs de Paris, Sorbonne Paris Cité, France

2 INFN, Sezione di Bologna and Dipartimento di Fisica e Astronomia dell'Università, Viale Berti Pichat 6/2, I-40127 Bologna, Italy
}

36th International Cosmic Ray Conference -ICRC2019-

July 24th - August 1st, 2019

Madison, WI, U.S.A.

\footnotetext{
*for collaboration list see also http://antares.in2p3.fr/Collaboration/index2.html

${ }^{\dagger}$ Speaker.
} 


\section{Introduction}

The main goal of the ANTARES neutrino telescope, a large-volume Cherenkov detector located under the Mediterranean Sea, 40-km off-shore Toulon, France, is to detect high-energy cosmic neutrinos [1]. High-energy neutrinos are produced in the decay chains of the products of cosmic ray interactions in the source, its surroundings or as they travel through the Universe. A diffuse flux can originate from the ensemble of unresolved individual sources, too faint to be detected, by cosmic ray propagation effects, or by a combination of these processes.

A highly-significant excess of high-energy neutrino events has been observed in data collected by the IceCube Neutrino Observatory. This excess is, so far, compatible with the hypothesis that this flux is isotropically distributed over the whole sky, in equipartition between the three neutrino flavours. This flux can be described by unbroken single-power-law energy spectra (i.e. $\mathrm{d} \Phi / \mathrm{dE} \propto$ $\mathrm{E}^{-\Gamma}$ ). Three main analyses have been carried out by the IceCube Collaboration: the High Energy Starting Events (HESE) search [2], the search for upward-going muons from the Northern Sky [3] and the all-sky combined analysis [4].

Some tension is present between the results obtained by the different IceCube analyses. The HESE search reports the observation of a rather soft energy spectrum $(\Gamma \simeq 2.9 \pm 0.2)$ as best-fit option for the cosmic signal. The analysis of upward-going muons from the Northern Sky finds the signal to be compatible with a hard energy spectrum $(\Gamma \simeq 2.1 \pm 0.1)$. The HESE cosmic signal is statistically dominated by cascade-like events at energies between 50 and $200 \mathrm{TeV}$, mostly coming from the Southern sky. The upwarg-going muons sample in IceCube is most sensitive above a few hundreds of $\mathrm{TeV}$, and only contains events coming from the Northern sky. The difference in the energy range of two analyses comes from the different atmospheric backgrounds of the two samples, being the atmospheric background in the cascade sample - i.e. a sample dominated by $v_{e}$ interactions - lower in normalisation than the one for upward-going events induced by $v_{\mu} \mathrm{CC}$ interactions.

Multiple-component hypotheses can explain this tension [5]. It has been argued that the Southern sky features the presence of the inner region of the Galactic plane, where soft-spectra neutrino sources could present, both as individual emitters (mainly Supernovae Remnants) and as the diffuse emission region induced by cosmic rays propagating in the dense interstellar medium in the inner Galactic plane. On the contrary, hard-spectra sources (such as blazars [6]) are expected in the extra-galactic fields, which dominate the Northern hemisphere. The contribution of the inner Galactic Plane diffuse neutrino emission to the all-sky IceCube neutrino signal has been severely constrained by an ANTARES-IceCube joint search [7].

ANTARES data offer the opportunity to perform a complementary search. The analysis of 2450 days of high-quality ANTARES data-taking time collected between 2007 and 2015 has provided the observation of a mild excess of data in the high-energy all-flavour neutrino data-sample with respect to the expected background from terrestrial atmospheric backgrounds [8]. That analysis has been updated adding to the data sample 880 days of data-taking time acquired by ANTARES between January 2016 and June 2018, and the new results are presented here. 


\section{ANTARES diffuse flux analysis}

The search for an all-sky diffuse flux of cosmic neutrinos strongly relies on the proper estimation of the atmospheric backgrounds by means of Monte Carlo simulations. This background can be decomposed into three components, all coming from the decay chains of cosmic rays interaction products in the atmosphere: atmospheric muons, conventional atmospheric neutrinos and prompt atmospheric neutrinos.

The flux of atmospheric muons provides the largest physical signal in a neutrino telescope, even though the large water overburden mildens its influence. It is, however, more than 4-5 orders of magnitude more intense than the atmospheric neutrino flux and $\sim 8$ orders of magnitude more intense than the cosmic signal. Thanks to the fact that only neutrinos can actually traverse the Earth, due to their small interaction cross section with matter, the whole Earth can be used as a shield against atmospheric muons, by looking at upward-going particles. Still, the signal produced by atmospheric muons can mimic that of upward-going particles: this residual contribution can be however reduced by means of an event selection based on the reconstruction quality parameters.

Once the atmospheric muon contribution is largely removed from the sample, only neutrinoinduced events survive. Atmospheric neutrinos are topologically indistinguishable in ANTARES from cosmic neutrinos. The only difference between background and signal neutrinos is indeed given by their energy spectrum. The conventional component [9] of the atmospheric neutrino flux, induced by the decays of pions and kaons in the atmosphere, is extremely soft $(\Gamma \simeq 3.5)$ [10]; the prompt component [11], induced by short-lived charmed mesons, is harder $(\Gamma \simeq 2.7)$ but still somewhat softer than the expected cosmic contribution $(\Gamma \simeq 2-2.5)$. The conventional atmospheric component is largely dominant in normalisation, while the prompt and the cosmic one are expected to be of comparable size. An energy reconstruction-related selection allows a statistical separation between the signal and the backgrounds.

The previously published ANTARES results [8] were obtained using data collected from 2007 to 2015 , for a total of 2450 days of data-taking time. In total 33 events were observed in data when $24 \pm 7$ were expected from the background. Specifically, the analysis covered both tracklike events, produced by $v_{\mu}$ CC interactions, and cascade-like events, coming from all-flavour neutrino interactions, the two samples being mutually exclusive. The track-like sample provided the observation of 19 events in data when 13.5 were expected from the background, while the cascade-like sample had 14 events observed in data over an expectation of 10.5 events.

The excess of events was fitted by means of a maximum likelihood procedure and the resulting signal flux had per-flavour normalisation at $100 \mathrm{TeV} \Phi_{0}(100 \mathrm{TeV})$ and spectral index $\Gamma$ equal to

$$
\begin{aligned}
& \Phi_{0}(100 \mathrm{TeV})=(1.7 \pm 1.0) \times 10^{-18}\left[\mathrm{GeV}^{-1} \mathrm{~cm}^{-2} \mathrm{~s}^{-1} \mathrm{sr}^{-1}\right] \\
& \Gamma=2.4_{-0.4}^{+0.5}
\end{aligned}
$$

while the atmospheric background was fitted to be $25 \%$ more intense than what is expected from the models used in the estimation. Systematics related to atmospheric background models and detector properties were considered in the result, as well. Overall, the significance of the excess was estimated to be of the order of $1.6 \sigma$ with the null-cosmic hypothesis rejected at $85 \%$ confidence level (c.l). 


\subsection{Event selection optimisation}

In order to estimate the optimally sensitive event selection, different event reconstruction quality parameters can be exploited along with the energy estimator. The best sets of cuts is estimated in the Model Rejection Factor (MRF) procedure [12], optimising for the best upper limit at $90 \%$ c.l. that can be put on the signal given the expected backgrounds. Since in this work data are added to an already available sample, the one of the previously published analysis, and considering this sample as already fixed, the MRF procedure has been modified. The best average upper limit at $90 \%$ c.1., $\bar{\Phi}_{90}$, as defined in the original MRF procedure, is here re-defined as

$$
\bar{\Phi}_{90}=\Phi_{\text {model }} \times \frac{\bar{s}_{90}\left(b_{2} \mid N_{o b s, 1}, \bar{N}_{b k g, 1}\right)}{s_{1}+s_{2}}
$$

where the subscript 1 indicates quantities coming from the previous results and subscript 2 the ones added in the new sample. For a defined number of simulated background events from the background estimation in the new dataset, $b_{2}$, an average over the $90 \%$ c.l. Feldman \& Cousins [13] upper limits, $\mu_{90}$, can be computed as

$$
\bar{s}_{90}\left(b_{2} \mid N_{o b s, 1}, \bar{N}_{b k g, 1}\right)=\sum_{k=0}^{\infty} \mu_{90}\left(k+N_{o b s, 1}, b_{2}+N_{b k g, 1}\right) \times \operatorname{Poisson}\left(k \mid b_{2}\right) .
$$

According to the MRF procedure, the event selection producing the best upper limit only depends on the spectral shape of the signal and not on its normalisation. An unbroken single power-law spectrum with spectral index $\Gamma=2.5$ has been chosen for the optimisation procedure. This value is compatible with our previous findings as well as consistent with the all-sky best fit hypothesis in IceCube data [4]. Given the low neutrino energy threshold of the detector - due to the spacing between the lines of the detector - ANTARES best performs for these soft spectra with respect to harder signals, especially if compared to the IceCube Neutrino Observatory.

The event selection has been optimised separately for the track-like and the shower-like event samples. In the case of track-like events, upward-going muon tracks are selected, requiring a high reconstruction quality in terms of the likelihood of the track fit, as well as requiring a good estimated angular resolution - which is not strictly required in high-energy diffuse searches, but has been proven to be effective in rejecting atmospheric muon events [14]. Finally, a statistical separation between atmospheric and cosmic events is obtained by means of a selection on the energy estimate for track-like events coming from an Artificial Neural Network (ANN) algorithm [15].

Events not entering the track-like sample are at first considered for the selection of shower-like events; because of this, the two samples are completely disjoint. A cylindrical fiducial volume is then defined, having a $300 \mathrm{~m}$ radius and $500 \mathrm{~m}$ height, centred at the baricenter of the instrumented volume. Only events having their reconstructed shower vertex within this volume are considered. The same pre-selection chain presented in [16] is then followed, and the final muon background rejection is done on the basis of a Random Decision Forest algorithm (RDF) and a muon-to-shower Likelihood Ratio test based on the hit patterns in the detector. Finally, the statistical separation between atmospheric and cosmic events is done on the basis of the reconstructed shower energy from the algorithm described in [17]. 


\section{Results}

The data sample considered in this update covers the period from January 2016 to June 2018, for a total of 880 days of detector data acquisition time. The analysis follows the data-blinding rules of the Collaboration: a limited set of data can be used only to check the agreement of data and Monte Carlo, and the optimisation of the event selection is based only on Monte Carlo simulations. These Monte Carlo simulations [18] reproduce the time-dependent data-taking conditions and the development of the efficiency of the detector [19] Once the optimised selection is applied to the Monte Carlo sample of the $\sim 900$ additional days, 6.4 background events are expected from atmospheric neutrinos interacting and producing track-like events above the energy cut value. This sample is extremely pure, with a percent-level contamination from atmospheric muons. When considering shower-like events, 5.7 background events are expected, with a 30\% contamination from atmospheric muons. After unblinding, 8 and 9 events are observed in the track and shower sample, respectively. Summing up this to the results obtained in the previously published analysis, a total of 50 high-energy events is present in data, when $36.1 \pm 8.7$ events are expected from the simulations of the background. The energy estimator distributions for the track-like and shower-like events are shown in figure 1.
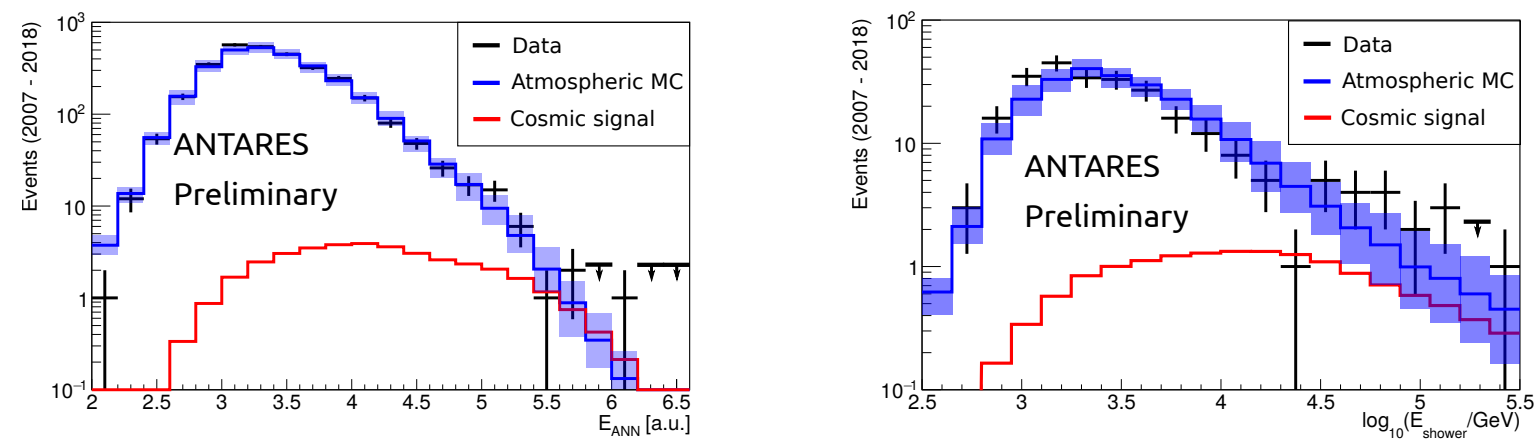

Figure 1: Left: energy estimator distribution for the track channel after the event selection chain. Right: same for the shower channel. Red lines show the cosmic neutrino expectation for the best-fit cosmic signal extracted from the ANTARES data-sample analysed here, while blue lines represent the contribution from atmospheric events, with the shaded areas providing and estimate of the related uncertainties.

The selection procedure produces a sample that contains the $1 \%$ highest-energy events observed by ANTARES. This roughly corresponds to events having a neutrino energy above $40 \mathrm{TeV}$. The observed event rate in data allows to exclude at $90 \%$ c.l., once systematics effects are taken into account according to the method of [20], the non-existence of a cosmic flux with normalisation similar to that observed in the IceCube samples [4].

The observed distributions of the energy estimators, after the final selection, are fitted using a maximum-likelihood method as done in [4] and [8]. Monte Carlo simulations are used to create templates of the cosmic signal and of the atmospheric backgrounds, considering different normalizations and spectral indexes for the signal. Binned distributions of energy estimators of data and of the Monte Carlo templates are considered. The final likelihood $L$ is given by the product of the individual likelihoods $L_{i, S}$ computed for each bin $i$ of the energy estimator distribution for the shower $(s h)$ and track $(t r)$ samples $S$ separately. The distribution of data and templates are compared con- 
sidering Poisson statistics, with a Gaussian penalty factor to account for systematic effects on the Monte Carlo input parameters $\tau_{j}^{*}$ :

$$
\begin{aligned}
L & =\prod_{S \in\{s h, t r\}} \prod_{i=0}^{N_{S}} L_{i, S} \\
L_{i, S} & =e^{-\mu_{i, S}} \cdot \frac{\mu_{i}^{k_{i, S}}}{k_{i, S} !} \cdot \prod_{j} \frac{1}{2 \pi \sigma\left[\tau_{i, S, j}\right]} e^{-\frac{\left(\tau_{i, S, j}-\tau_{i, S, j}^{*}\right)^{2}}{\sigma^{2}\left[\tau_{i, S, j}\right]}}
\end{aligned}
$$

where $\mu_{i}$ is the expected number of events in the $i$-th bin from the simulated templates, $N_{S}$ is the number of bins in the energy estimator histogram for each event sample and $k_{i, S}$ is the number of events observed in data for that event sample in that bin. The nuisance parameters $\tau_{i, S, j}$ considered here are: the atmospheric neutrino background normalization; the residual atmospheric muon background, relevant in the shower analysis only; an energy-scale shift, which can be produced by the uncertainty on water properties and optical module efficiencies, as well as on the response of the PMTs. The considered effects modify the expected $\mu_{i}$ in the simulated templates analogously to what has been reported above. The atmospheric normalization is fitted simultaneously for the two samples, assuming that the background fluxes should follow the same modeling. The possible energy shift is considered separately for cascades and tracks. The 2D log-likelihood profile, after having fixed the nuisance parameters to the best-fit values, is shown in figure 2 . The $68 \%$ and $90 \%$ c.l. contours from this analysis are shown in the plot. The resulting best-fit parameters for the per-flavour cosmic flux normalisation and spectral index are

$$
\begin{aligned}
& \Phi_{0}(100 \mathrm{TeV})=(1.5 \pm 1.0) \times 10^{-18}\left[\mathrm{GeV}^{-1} \mathrm{~cm}^{-2} \mathrm{~s}^{-1} \mathrm{sr}^{-1}\right] \\
& \Gamma=2.3_{-0.4}^{+0.4}
\end{aligned}
$$

The significance of this excess over the background-only hypothesis is of $1.8 \sigma$, estimated by means of a likelihood-ratio test between the best-fit point and the null-cosmic flux outcome.

If the maximum likelihood procedure is performed separately for track-like and shower-like events, the best-fit parameters are

$$
\begin{aligned}
\Phi_{0, t r}(100 \mathrm{TeV}) & =\left(0.8_{-0.6}^{+0.5}\right) \times 10^{-18}\left[\mathrm{GeV}^{-1} \mathrm{~cm}^{-2} \mathrm{~s}^{-1} \mathrm{sr}^{-1}\right] \\
\Gamma_{t r} & =2.0_{-0.4}^{+0.8}
\end{aligned}
$$

and

$$
\begin{aligned}
\Phi_{0, s h}(100 \mathrm{TeV}) & =(2.1 \pm 0.8) \times 10^{-18}\left[\mathrm{GeV}^{-1} \mathrm{~cm}^{-2} \mathrm{~s}^{-1} \mathrm{sr}^{-1}\right] \\
\Gamma_{s h} & =2.4_{-0.4}^{+0.4}
\end{aligned}
$$

for tracks and showers respectively. The different normalisation and spectral indexes can be attributed to the fact that the separate fit produces also different normalisation factors for the atmospheric components. Indeed, in the track channel, the atmospheric contribution is estimated to be about 30\% higher than the models, while in the case of shower-like events the best-fit is for an atmospheric flux compatible with the predictions. 


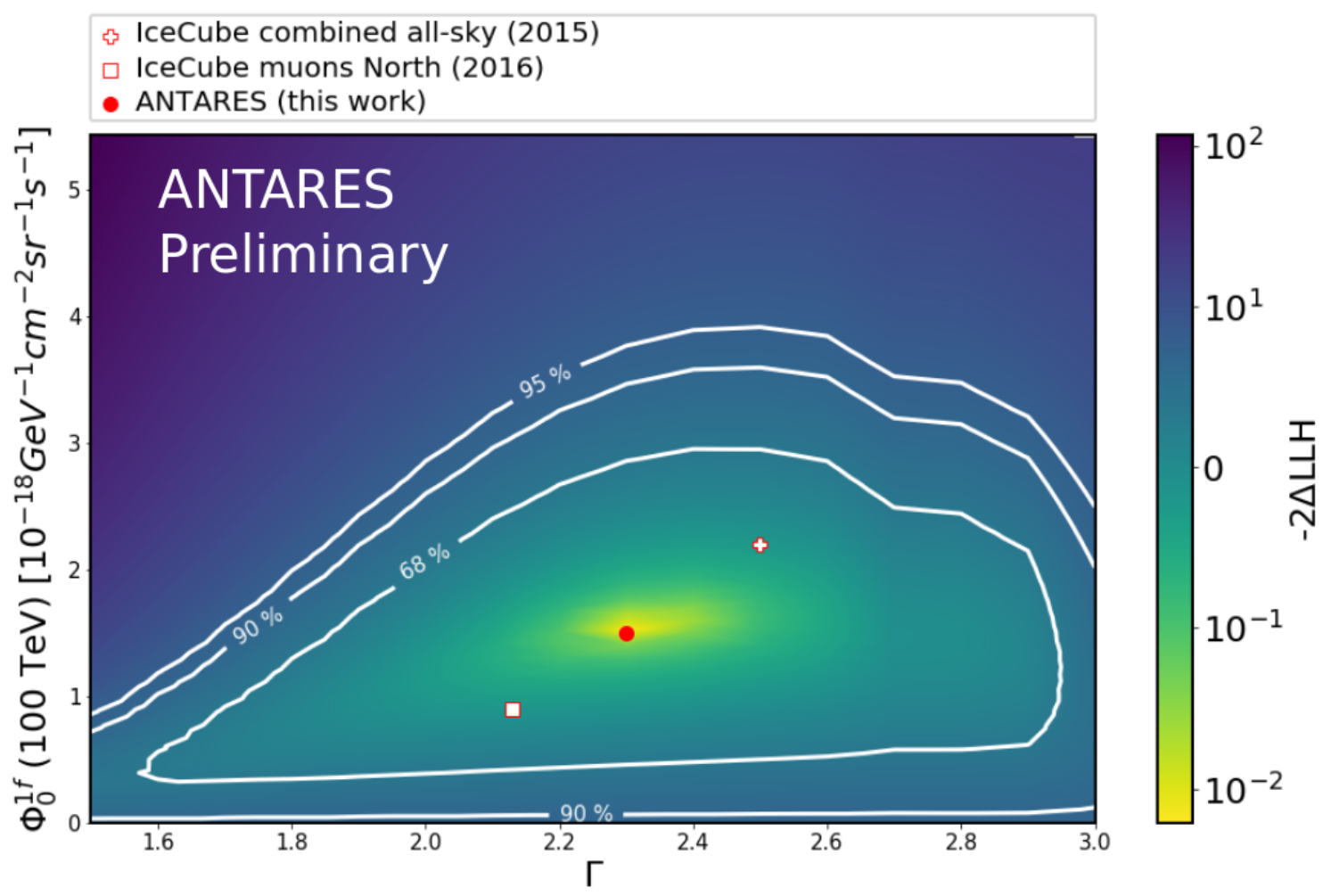

Figure 2: 2D likelihood landscape of the parameter fitting procedure described in the text for the spectral index $\Gamma$ and the one-flavour flux normalisation at $100 \mathrm{TeV} \Phi_{0}(100 \mathrm{TeV})$; the ANTARES best fit point from this work is shown as a red circle. Also shown the IceCube combined all-sky best fit from ref. [4] (empty cross) and the IceCube result from analysis of Northern-sky upward-going muons [3]. The while countours show the $68 \%, 90 \%$ and $95 \%$ confidence areas.

Being the track sample more pure and with higher statistics, it can be used to perform a further analysis of the atmospheric components. In particular, the energy distribution at the detector is dominated by atmospheric neutrinos below a few $\mathrm{TeV}$, where the conventional component is dominant. Using these lower-energy events - those with the ANN energy estimator below 4.4 in figure 1-left - the spectral behaviour of the conventional component can be constrained. The prediction from ref. [9] have been tested. The normalisation and a tilt in the spectral shape have been considered in the likelihood procedure, producing a best fit normalisation equal to $1.30 \pm 0.05 \times \Phi_{\text {model }}$, with a spectral index deviation $\Delta \Gamma_{H o n d a}=-0.1 \pm 0.1$.

\section{Outlook}

A new event selection in the shower-like sample, which will allow a better handling of the atmospheric muon contamination, as well as an improvement in the statistics of shower-like events is currently under development. This will allow a better determination of the atmospheric component also in this channel, thus a better understanding of the high-energy flux. In particular, the combined information from the shower and track samples could allow constraining the prompt atmospheric contribution. In addition, an updated simulation of the detector response will improve 
the agreement between data and Monte Carlo in the energy estimation of shower-like events, which will also provide a better estimation of the atmospheric neutrino background. It is foreseen that, all these factors taken into account, the possibility to study the diffuse cosmic neutrino signal with ANTARES will be further improved in the future.

\section{Acknowledgements}

This project has received funding from the European Union's Horizon2020 research and innovation programme under grant agreement $\mathrm{N}^{\circ} 739560$ (KM3NeT 2.0)

\section{References}

[1] R. Coniglione on behalf of the ANTARES and KM3NeT Collaborations, PoS(ICRC2019)006

[2] M. G. Aartsen et al., Physical Review D 91 (2015) 022001

[3] M. G. Aartsen et al., Astrophysical Journal 833 (2016) 3

[4] M. G. Aartsen et al., Astrophysical Journal 809 (2015) 98

[5] A. Palladino and W. Winter, A multi-component model for observed astrophysical neutrinos, Astronomy \& Astrophysics 615 (2018) A168

[6] M.G. Aartsen et al., Science 361 (2018) 147-151

[7] A. Albert et al., Astrophysical Journal Letters 868 (2018) L20

[8] A. Albert et al., Astrophysical Journal Letters 853 (2018) L7

[9] M. Honda et al., Physics Review D 75 (2007) 043006

[10] S. Adrián Martínez et al., European Physics Journal C 73 (2013) 2606

[11] R. Enberg, M.H. Reno and I. Sarcevic, Physics Review D 78 (2008) 043005

[12] G.C. Hill and K. Rawlins, Astroparticle Physics 19 (2003) 393

[13] G.J. Feldman and R.D. Cousins, Physics Review D 57 (1998) 3873

[14] S. Adrian-Martinez et al., Astrophysical Journal 760 (2012) 53

[15] J. Schnabel et al., Nuclear Instruments and Methods in Phyics A 725 (2013) 106

[16] A. Albert et al., Physics Review D 96 (2017) 082001

[17] A. Albert et al., Astronomical Journal 154 (2017) 275

[18] L.A. Fusco and A. Margiotta, on behalf of the ANTARES Collaboration, EPJ Web Conf. 116 (2016) 02002

[19] A. Albert et al., European Physics Journal C 78 (2018) 669

[20] J. Conrad et al., Physics Review D 67 (2003) 012002 